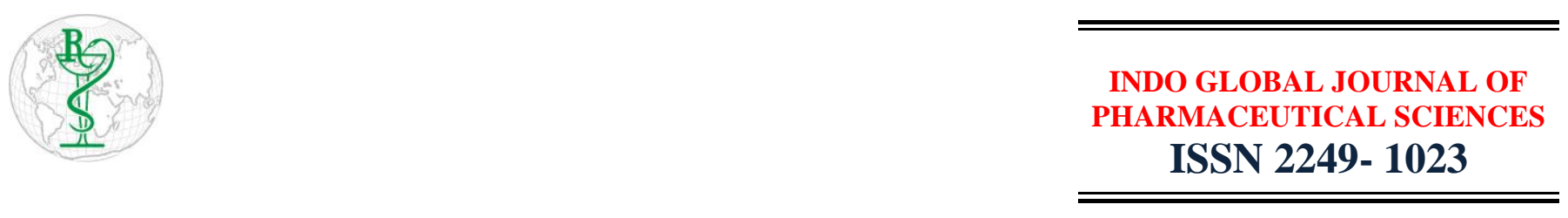

\title{
In Sighting Role of Ashwagandha: Pharmacological Review
}

\begin{abstract}
Received:
01.03.2019

Accepted:

25.03.2019

Keywords

Withania

somnifera;

Withanolides;

Sitoindoside;

Antioxidant;

Anti-arthritis;

Macrophages.
\end{abstract}

\author{
Hardeep Singh *1, Pooja Sharma ${ }^{1,2}$, Dinesh Kumar ${ }^{1}$ \\ ${ }^{I}$ Sri Sai College of Pharmacy, Manawala, Amritsar-143115, Punjab, India \\ ${ }^{2}$ Department of Pharmaceutical Sciences and Drug Research, Punjabi University Patiala, Punjab, India
}

Address for Correspondence: Hardeep Singh; hardeep770099@gmail.com

\begin{abstract}
Withania somnifera (Solanaceae), commonly known as Ashwagandha is an important medicinal plant that has been used in Ayurvedic medicines for more than 3,000 years. It is specially used for the treatment of nervous and sexual disorders. The chief active chemical constituents are withanolides, withaferin $\mathrm{A}$, withaniferin $\mathrm{B}$, withanine, somniferine, somnine, sitoindoside. Withanolides are used in the treatment of arthritis and are shown to be potent inhibition of the activation of NF-kB. Withanone protects against the cerebral damage. It also exhibits antioxidant effects by increasing the antioxidant enzymes. Sitoindosides enhance the M1 and M2 muscarinic receptor binding to alter the AChE activity. Withania somnifera also showed significance protection against stress induced gastric ulcers. Withania somnifera showed a wide range of therapeutic activities and maintaining general health such as Immunomodulation, anti-cancer and anti-epileptic, memory enhancer, to promote good physical and mental health, mood elevator, diuretic, general tonic and rejuvenator, stress reliever, anti-ageing, anti-oxidant, hypoglycemic, hypocholesterolemic. Withanolides have got analgesic and anti-inflammatory activity due to cyclooxygenase- 2 inhibition property. Ashwagandha enhances nitric oxide synthase activity of the macrophages, which in turn increases the microbial killing power of these immune cells thereby enhancing the Cell Mediated Immune (CMI) response. This plant is used due to its wide range of therapeutic activities and lesser side effects as compare to synthetic drugs. () 2019 iGlobal Research and Publishing Foundation. All rights reserved.
\end{abstract}

Cite this article as: Singh, H.; Sharma, P.; Kumar, D. In Sighting Role of Ashwagandha: Pharmacological Review. Indo Global J. Pharm. Sci., 2019; 9(2Suppl.): 145. DOI: http://doi.org/10.35652/IGJPS.2019.92S43 .

Indo Global Journal of Pharmaceutical Sciences( ISSN 2249 1023; CODEN- IGJPAI; NLM ID: 101610675) indexed and abstracted in CrossRef (DOI Enabling), UGC CARE Journal List, EMBASE(Elsevier), National Library of Medicine (NLM) Catalog, ResearchGate, Publons, CAS (ACS), Index Copernicus, Google Scholar and many more. For further details, visit http://iglobaljournal.com

This is a special issue as an outcome of 'RAPSCON-2019' sponsored by APTI and organized by Sri Sai College of Pharmacy, Manawala, Amritsar, Punjab, India. Relaxation offered in journal format. 